\title{
Evidence of High-Detail Perceptual Modeling in the Visual Cortex
}

\author{
Terry B. Bollinger (1) 1 \\ ${ }^{1}$ Apabistia Research, Ashburn, VA, USA 20147
}

(Incepted 20 January 2020; Published 11 July 2021; Tweaked 2021-07-11.11:52)*

\begin{abstract}
This informal but well-referenced description of an afterimage experiment called Ghost Tap provides persuasive and easily reproducible evidence that the visual cortex plays a significant role in certain classes of long-duration visual afterimages. Subjects of the experiment literally cannot discern the difference between the afterimage and reality, resulting in easy startling of the subjects when physical motion in the room no longer matches the persuasive afterimages they are perceiving. Anecdotal examples of less extreme versions of the same effect suggest that the Ghost Tap effect has, over centuries, intentionally and unintentionally helped persuade people of the existence of nominally "supernatural" effects that are just persuasive long-duration afterimages. While this description is informal, the easy reproducibility of the Ghost Tap makes it a good candidate for more precise and quantitative studies. One theory why Ghost Tap exists is that it is part of load reduction and speed enhancement strategy to compensate for the slow processing speeds of neurons. Maintaining a dynamic and predictive real-time model of likely sensory inputs from the external world would enable the brain to discard quickly and with minimal processing any sensory inputs that fall within the predictive tolerance limits to the current model state. A perceptive load reduction interpretation of the Ghost Tap argues that the ability of the brain to support dreaming in vivid detail is likely a direct corollary of its ability to create dream-like waking states for faster and more efficient processing of large sensory loads. If the brain regularly uses dream-like waking states to reduce data, more study of effects like Ghost Tap might help explain the frequency of pathologies in which perception becomes disconnected from reality.
\end{abstract}

DOI: https://doi.org/10.48034/20200120
This is about: Perceptive modeling, Positive afterimages, Filled-in representation, Cortical representation, Perceptive load reduction

Published by Apabistia Press under the Creative Commons Attribution 4.0 International License. You are free to redistribute this article as you choose, provided only that you preserve the author attribution, article title, journal citation, and DOI.

${ }^{*}$ This document uses Kenobi versioning. The online name always points to the most recent document version, while obl, ob2,... suffixes indicate obsolete releases of the document. 


\title{
The Ghost Tap: A Demo of Positive Afterimages in the Visual Cortex
}

\author{
Terry Bollinger \\ terrybollinger@gmail.com \\ 2020 Jan 20 (Quora Post Time) \\ Includes minor edits and type fixes. The original post is located at: \\ https://www.quora.com/Can-you-tell-me-a-creepy-psychological-trick/answer/Terry-Bollinger
}

\section{Quora Title: Can you tell me a creepy psychological trick?}

\section{Quora Author: Terry Bollinger}

This trick is not just creepy, it can be scream-out-loud terrifying. Please use caution.

To be precise, it is more of a neural perception trick than a psychological trick. It is a stronger, more repeatable, and multi-person version of the same trick that Aishah Hannan described in another answer, with a couple of twists.

[2020-01-22 Addendum: Henrique Kenzo also describes it! So, it's called "Ver a Matuto" in Brazil. That is fascinating, I've never seen a name for it before! I guarantee you my version is both easier and scarier, though, and there's no need to set your alarm at 3am.]

I call it The Ghost Tap. If you try it, please be both cautious and ethical. Do not do it to anyone with a relevant medical condition, even if you tell them up front what is really going on. I recommend that you either explain what is really going on (it will still frighten them), or at the very least begin with an up-front disclaimer that no matter what you may say next, this is a magician's trick, and what they are about to experience is not real.

So here's The Ghost Tap:

(1) Get some kind of easily triggered flash device, such as a cell phone camera set on flash mode.

(2) Go into a completely dark room, such as a large closet, with a friend or two (or a large mirror if you prefer looking only at yourself).

(3) Wait for at least two minutes until your eyes adjust to the dark.

(4) This part is important: Tell everyone that they must stay absolutely still for what comes next, and then for a couple of minutes afterwards. It is especially important that everyone keep their heads still and their eyes focused on the same spot. Relaxing can accidentally produce the needed stillness (as in the Aishah Hannan version), but the effect is independent of your mental state. It does not matter if you are relaxed, tense, or freaking out. It only matters that you don't move!

(5) With as little motion as possible, click the flash.

(6) The immediate result will be a second or so of a pulsing negative afterimage. Everyone must just stay frozen in place and wait. 
(7) Here, finally, is the fun part: Over the next few seconds, if you succeed in staying perfectly still, an exceptionally sharp positive afterimage of the scene will emerge. It will look exactly as if the flash has started glowing again, but this time in a soft, violet, continuous light that erases all color, but clearly and sharply illuminates the scene.

(8) It's all an illusion!

As best I've been able to determine (I have never uncovered any papers on it), this crisp, positive, violet-only afterimage is a neurological afterimage, rather than the more common retinal kind.

That is, the flash leaves a subtle temporary chemical signature on the nerves and possibly brain cells involved in interpreting vision. This signature is likely both very common and quite mild. It is usually ignored by the brain, which is very good at intelligently ignoring deceptive sensory inputs. (It has to be, since if it was not, the result would be either hallucination or psychosis.) However, in the absence of further light plus the complete absence of head or eye motion, that same very smart brain finally decides that the mild signals are real sensory inputs after all. So it decides to present them to our consciousness as real, exceptionally convincing images. Unlike retinal afterimages, these neural afterimages benefit from the full "dressing up" that the brain provides to anything it considers to be fully validated sensory inputs.

(9) The vivid positive afterimage will linger for several seconds, and there are all sorts of fun (and terrifying) games you can play while it is active.

The other people cannot see you in the dark, but their brains will tell them that you are standing right there, completely immobile just like them. So imagine how they will react if you invisibly reach out and tap them on the shoulder...: :)

If and only if you follow magician's brotherhood ethical rules and warn everyone up front that what follows is only a magician's illusion, here's a good magician's patter (talk to misdirect attention) to use:

"Most folks don't know this, but some forms of bright flashes also emit a special violet afterglow for a few seconds afterwards. Now, what's amazing about this afterglow is that ghosts can also see it very clearly, and come rushing to see what is going on. Often they come in so fast that they break through the usual barriers and end up bumping into our world. So, please be ready just in case that happens to you. They mean you no harm, but the bumps from hurrying ghosts can be quite startling."

So that's it. Again, this is a delightfully unexpected and creepy effect, but one that is experimentally accessible and firmly based in the realities of both chemistry and neurology. In fact, if someone reading this is looking for a good experimental thesis topic in neurology, this concept of neural afterimages might be a good area to look into. The last time I checked many years ago, it did not seem to be a well-known effect at all.

2020-10-01 Addendum: While creating this Archive version of my (quite popular, hundreds of thousands of reads) Quora posting on the Ghost Tap, I came up with a couple of search phrases, "positive afterimages" and "long-lasting afterimages", that enabled me at last to find some relevant references. This remains an understudied area, however, which I persistently find surprising given that it seems to provide some rather striking insights into how the brain models reality.

The older 1916 article [1] that I found, by P. F. Swindle of Ohio State University, includes studies of positive afterimages in both birds (pp. 324-329) and humans (pp. 329-334). Swindle explores several intriguing variations, and remarks several times about the importance of avoiding rapid motion to view the positive after images. Interestingly, Swindle's positive afterimages are not pale violet, but in full and even vivid color. I suspect my violet color results from using an intensely bright flash that overloads retinal color receptors. He observes in the final paragraph (p. 334) that positive afterimages can be invoked minutes after viewing a scene. He speculates that spiritualists are aware of this effect, and use of it to persuade people they are seeing ghosts. 
The more recent 2004 abstract-only article by Alex O. Holcombe et al [2] focuses instead on a very narrow situation in which they show that positive afterimages cannot be retinal, but must be the result of a persistent "cortical" filling in of a simple image that has been retained by the brain in the absence of new conflicting visual data. The authors assert in that their results "reflect the persistence of a [cortical] visual representation in the absence of evidence that the world has changed." While no paper was ever written to fill out the details of this abstract, the authors did give a detailed poster session [3] on the same work (Figure 1).

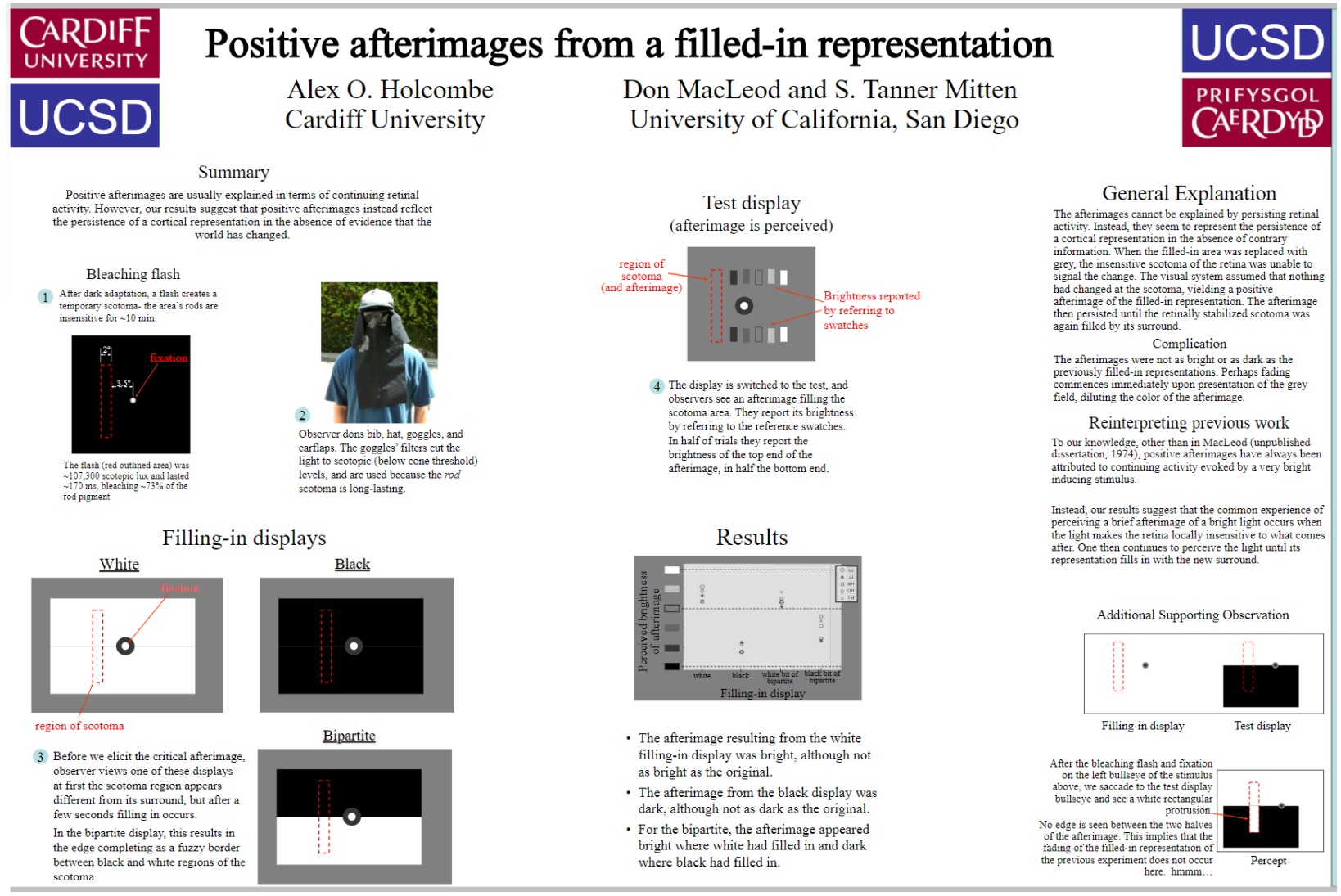

FIG. 1. Positive afterimages from a filled-in representation [Poster]

The Holcombe et al. interpretation appears to support the view that the positive image results from persistent models in the brain, both to enable interpretation of images and allow faster reduction of new visual data.

There are many more interesting papers relevant to this topic. In order from most recent to oldest and including diverse interpretations of such phenomena, these include:

2020 Miller, Holcombe, and Latham [4]

2020 Robinson, Grootswagers, Shatek, Gerboni, Holcombe, and Carlson [5]

2019 Zenkin [6]

2017 Dong, Holm, and Bao [7]

2004 Holcombe, MacLeod, and Mitten [Abstract] [2]

2004 Holcombe, MacLeod, and Mitten [Poster] [3]

2002 Eagleman and Holcombe [8]

1986 Rushton and MacLeod [9]

1982 Adelson [10]

1981 Williams [11]

1974 MacLeod and Hayhoe [12]

1916 Swindle [1] 


\section{REFERENCES}

[1] P. F. Swindle, "Positive After-Images of Long Duration," The American Journal of Psychology, vol. 27, pp. 324-334, 1916.

[2] A. O. Holcombe, D. I. A. MacLeod and S. T. Mitten, "Positive afterimages caused by a filled-in representation [Abstract]," Journal of Vision, vol. 4, pp. 485-485, 2004.

[3] A. O. Holcombe, D. I. A. MacLeod and S. T. Mitten, "Positive afterimages caused by a filled-in representation [Poster]," Journal of Vision, 2004.

[4] K. Miller, A. Holcombe and A. J. Latham, "Temporal phenomenology: phenomenological illusion versus cognitive error," Synthese, vol. 197, p. 751-771, 2020.

[5] A. K. Robinson, T. Grootswagers, S. M. Shatek, J. Gerboni, A. Holcombe and T. A. Carlson, "Overlapping neural representations for the position of visible and imagined objects," NBDT, 2020.

[6] G. M. Zenkin, "The rich afterimage illusions under head-image tilting and the internal visual space model," New Frontiers in Ophthalmology, vol. 5, p. 1-10, 2019.

[7] B. Dong, L. Holm and M. Bao, "Cortical mechanisms for afterimage formation: evidence from interocular grouping," Scientific reports, vol. 7, p. 1-13, 2017.

[8] D. M. Eagleman and A. O. Holcombe, "Causality and the perception of time," Trends in cognitive sciences, vol. 6, p. 323-325, 2002.

[9] W. A. H. Rushton and D. I. A. MacLeod, "The equivalent background of bleaching," Perception, vol. 15, p. 689-703, 1986.

[10] E. H. Adelson, "The Delayed Rod Afterimage," Vision Research, vol. 22, p. 1313-1328, 1982.

[11] D. R. Williams, D. I. A. MacLeod and M. M. Hayhoe, "Punctate sensitivity of the blue-sensitive mechanism," Vision Research, vol. 21, p. 1357-1375, 1981.

[12] D. I. A. MacLeod and M. Hayhoe, "Rod Origin of Prolonged Afterimages," Science, vol. 185, p. 1171-1172, 1974. 